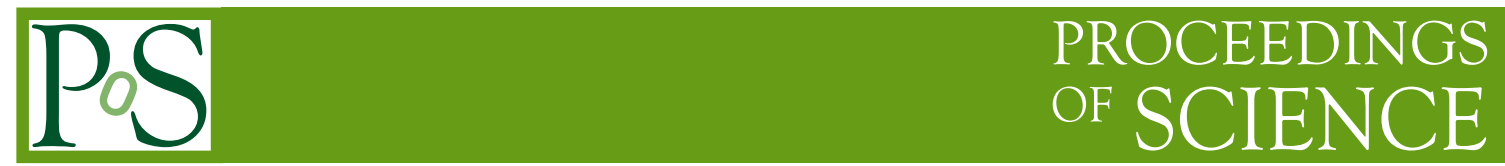

\title{
Highlights from CMS
}

\section{Ferenc Siklér* on behalf of the CMS Collaboration}

KFKI Research Institute for Particle and Nuclear Physics, Budapest, Hungary

E-mail: siklerermki.kfki.hu

The CMS experiment and its detector systems show an excellent performance. Many aspects, physics objects, are already commissioned. The collaboration produced pioneering results from a small data sample: the first publications using the 0.9 and $2.36 \mathrm{TeV}$ data are already available. This is just the beginning of a successful physics program at the LHC, with possible future discoveries.

XVIII International Workshop on Deep-Inelastic Scattering and Related Subjects, DIS 2010 April 19-23, 2010

Firenze, Italy

\footnotetext{
* Speaker.
} 


\section{Introduction}

The Compact Muon Solenoid (CMS) experiment has detected the first proton-proton collisions at the end of November last year. During this 2009 run more than 300 thousand minimum bias events were collected at $\sqrt{s}=0.9 \mathrm{TeV}$ and about 20 thousand at $2.36 \mathrm{TeV}$ (Fig. 1).

In the early spring of 2010 the experiment was running with a fully open minimum bias trigger, with typical rates of 50-100 Hz. Having seen the first $7 \mathrm{TeV}$ collisions on March 30th, there are several million collisions recorded so far, with very many ongoing analyses extracting physics information from the data.

This report shows the detector and reconstruction performance, as well as the first physics results produced in a very short time.

\section{Detector}

A detailed description of the CMS experiment can be found elsewhere [1]. The central feature of the CMS apparatus is a superconducting solenoid, of $6 \mathrm{~m}$ internal diameter. Within the $B=$ 3.8 $\mathrm{T}$ field volume are the silicon pixel and strip trackers, the crystal electromagnetic calorimeter (ECAL) and the brass-scintillator hadronic calorimeter (HCAL). Muons are measured in gaseous detectors embedded in the iron return yoke. Besides the barrel and endcap detectors, CMS has extensive forward calorimetry.

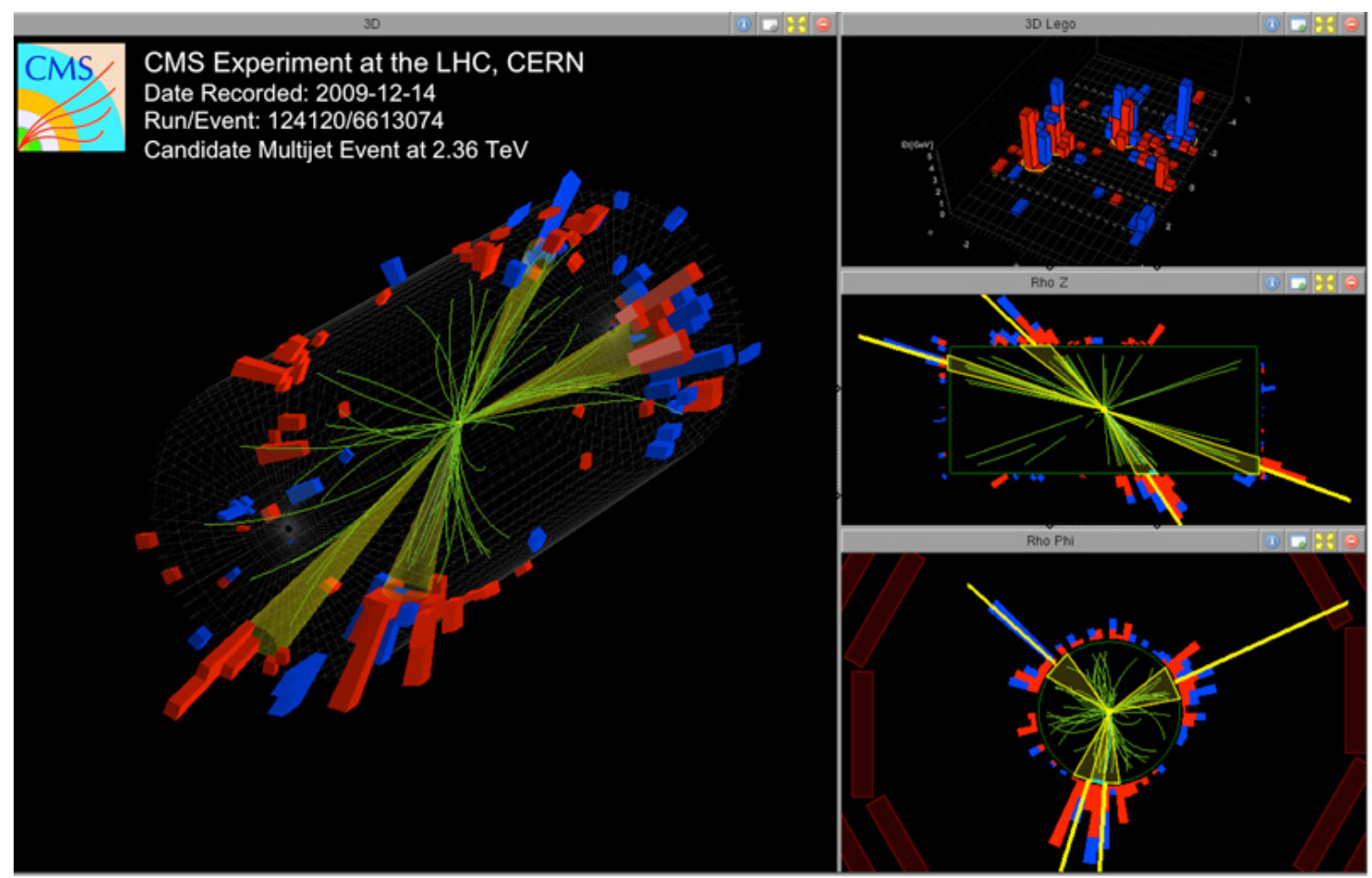

Figure 1: A reconstructed $2.36 \mathrm{TeV}$ proton-proton event with tracks and calorimeter towers. 

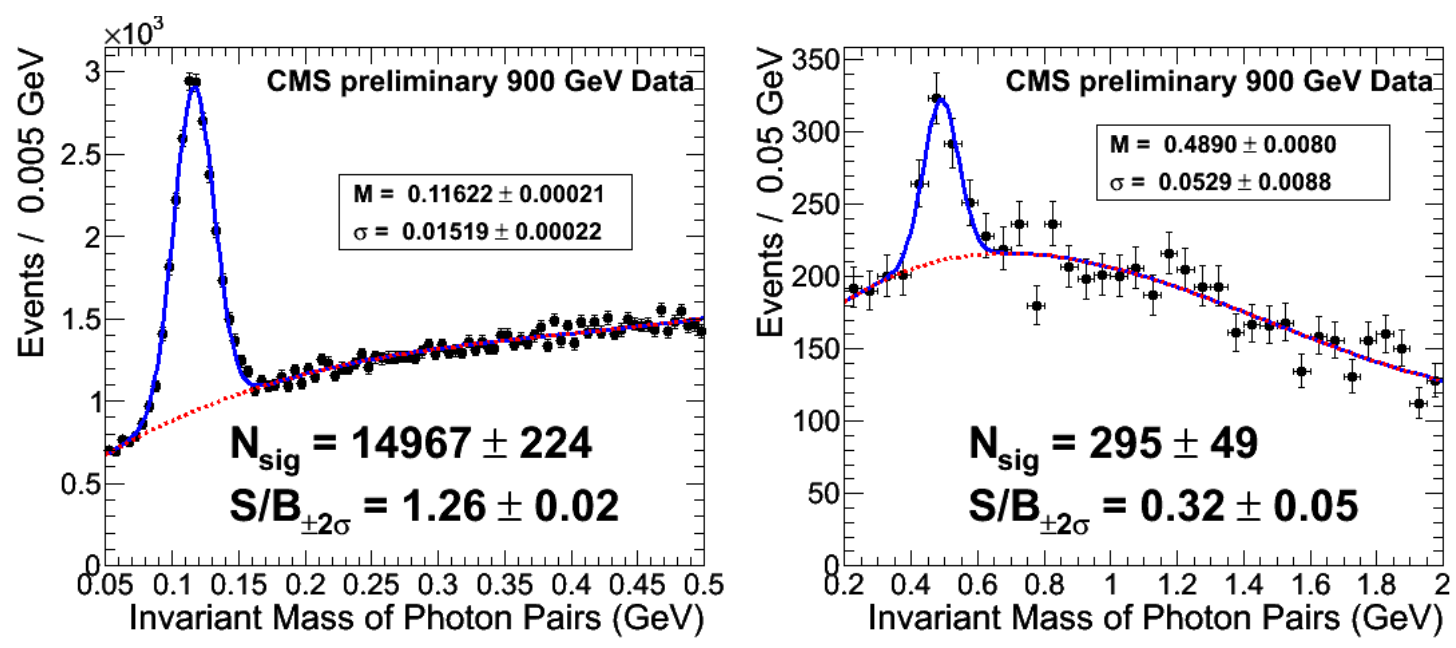

Figure 2: Left: Invariant mass spectrum of photon pairs in ECAL barrel with $E_{T}(\gamma)>300 \mathrm{MeV}$ and $E_{T}\left(\pi^{0}\right)>900 \mathrm{MeV}$, with uncorrected energies. Right: Invariant mass spectrum of photon pairs in ECAL barrel with $E_{T}(\gamma)>400 \mathrm{MeV}$ and $E_{T}(\eta)>2 \mathrm{GeV}$, obtained with $\pi^{0}$ veto and uncorrected energies.

Mid-rapidity charged particles are tracked by three layers of silicon pixel detectors, made of 66 million $100 \times 150 \mu \mathrm{m}^{2}$ pixels, followed by ten microstrip layers, with strips of pitch between 80 and $180 \mu \mathrm{m}$. The silicon tracker provides the vertex position with $\sim 15 \mu \mathrm{m}$ accuracy.

The ECAL has an energy resolution of better than $0.5 \%$ above $100 \mathrm{GeV}$. The HCAL, when combined with the ECAL, measures jets with a resolution $\Delta E / E \approx 100 \% / \sqrt{E} \oplus 5 \%$. The calorimeter cells are grouped in projective towers, of granularity $\Delta \eta \times \Delta \phi=0.087 \times 0.087$ at central rapidities and $0.175 \times 0.175$ at forward rapidities. The lead-tungstate crystals are $25.8 X_{0}$ thick in the barrel and $24.7 X_{0}$ thick in the end-caps.

The muons are measured in the pseudorapidity window $|\eta|<2.4$, with detection planes made of three technologies: Drift Tubes, Cathode Strip Chambers, and Resistive Plate Chambers. Matching the muons to the tracks measured in the silicon tracker results in a transverse momentum resolution between 1 and $5 \%$, for $p_{\mathrm{T}}$ values up to $1 \mathrm{TeV} / c$.

The very forward angles are covered by the CASTOR $(5.3<|\eta|<6.6)$ and Zero Degree $(|\eta|>8.3)$ calorimeters. They are made of quartz fibers/plates embedded in tungsten absorbers.

\section{Performance}

The invariant mass distributions of photon pairs detected in ECAL barrel are shown in Fig. 2, for $\pi^{0}$ and $\eta$. The plots do not yet contain corrections for shower containment, thresholds and energy loss. The peak position and the ratio of signal to background show a good agreement between data and simulation. The energy scale is also seen to agree within $2 \%$.

Electrons are reconstructed and identified with the combination of tracker and ECAL information. The observed $p_{T}$ spectrum shows a good agreement with simulation [2]. Photon conversions are also reconstructed, this way one can even study $\pi^{0}$ particles where one decay photon was converted in the tracker [2]. 

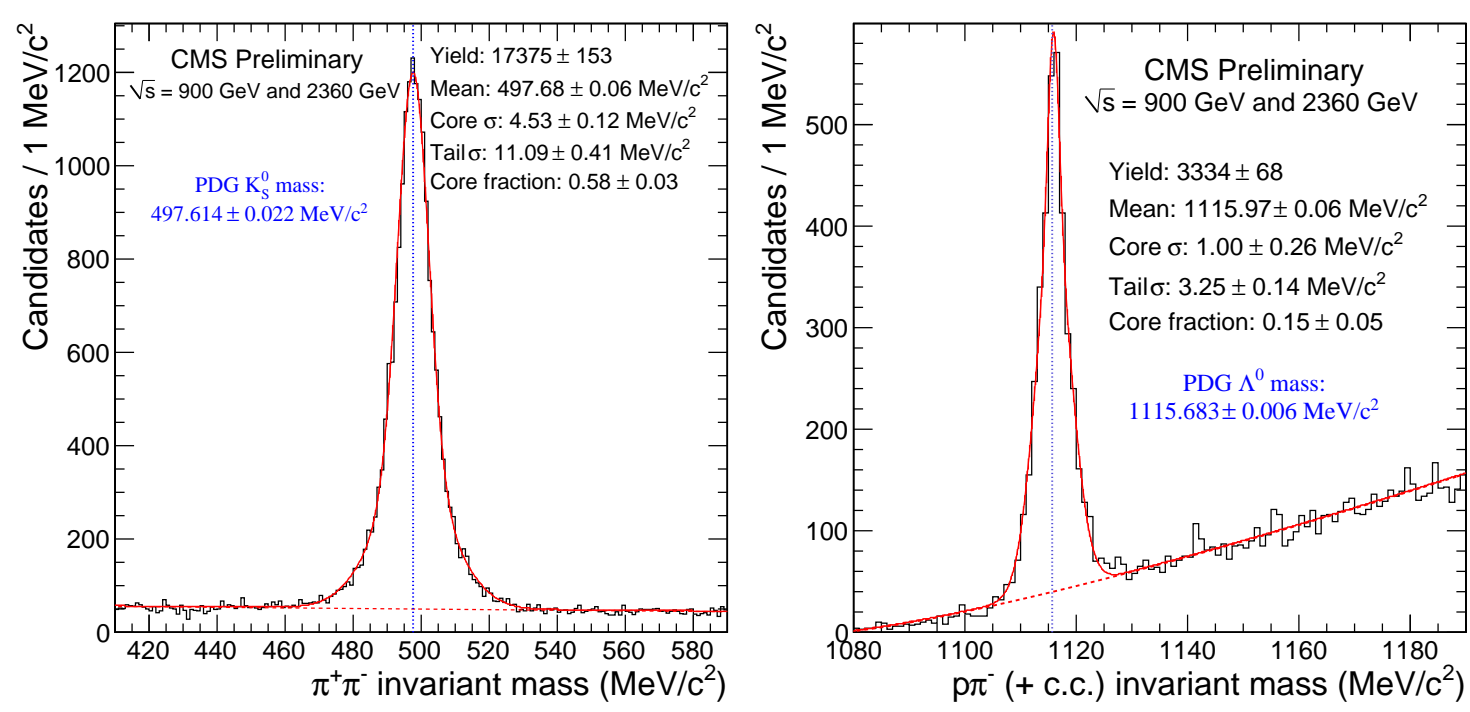

Figure 3: Left: Fitted $\pi^{+} \pi^{-}$mass spectrum for data. Right: Fitted $\mathrm{p} \pi^{-}$(+ charge conjugate) mass spectrum for data. Uncertainties shown are statistical only.

The performance of tracker is demonstrated with the help of long lived resonances, decaying to charged hadrons off the primary interaction vertex [3]. In Fig. 3 invariant mass spectra of $\pi^{+} \pi^{-}$pairs, as well as and $\mathrm{p} \pi^{-}\left(\overline{\mathrm{p}} \pi^{+}\right)$pairs are shown with fits to signal and background. The obtained fit parameters match very well the mass of $\mathrm{K}_{\mathrm{S}}^{0}$ and $\Lambda$, while the signal width and the shape of the background agree with simulation results.

The resonances identified can be combined with other charged hadrons to get to higher resonances, such as the on-vertex $\mathrm{K}^{*}(892)$ and the $\Xi^{-}\left(\bar{\Xi}^{+}\right)$. The mass peaks of both particles show an excellent agreement with literature numbers [3].

The tracker can be used for particle identification at low momentum, since the energy deposited in the silicon layers allows to measure the energy loss rate of the charged particle (Fig. 4-left), in this case with a power mean estimator [3]. That capability is readily used to enhance kaons in order to facilitate the yield extraction of the $\phi(1020)$ meson (Fig. 4-right).

The tracker will also be used to tag bottom jets [3]. The measured distribution of some relevant b-tagging related variables along with the expected light, charm and bottom jet contributions from simulation are shown in Fig. 5. The impact parameter significance is plotted for those tracks which have at least 7 hits, associated to a jet, with respect to the primary vertex. The distribution of the number of tracks at a secondary vertex shows the sensitivity to heavier jets, if 4 or more tracks are seen. In summary all the basic b-tagging variables are well described by simulation.

In order to extract and properly combine information from several subdetectors CMS adopted a new reconstruction approach, the so called particle flow method [4]. During reconstruction links between several objects (e.g. tracks and calorimeter clusters) are created based on their distance. The method shows an improved performance for photons, charged and neutral hadrons, jets and missing $E_{T}$. For example, the reconstructed $\pi^{0}$ mesons in the ECAL barrel region $(|\eta|<1.0)$ have correct mass position with a $13 \mathrm{MeV} / c^{2}$ resolution. It is also used to optimize energy resolution 

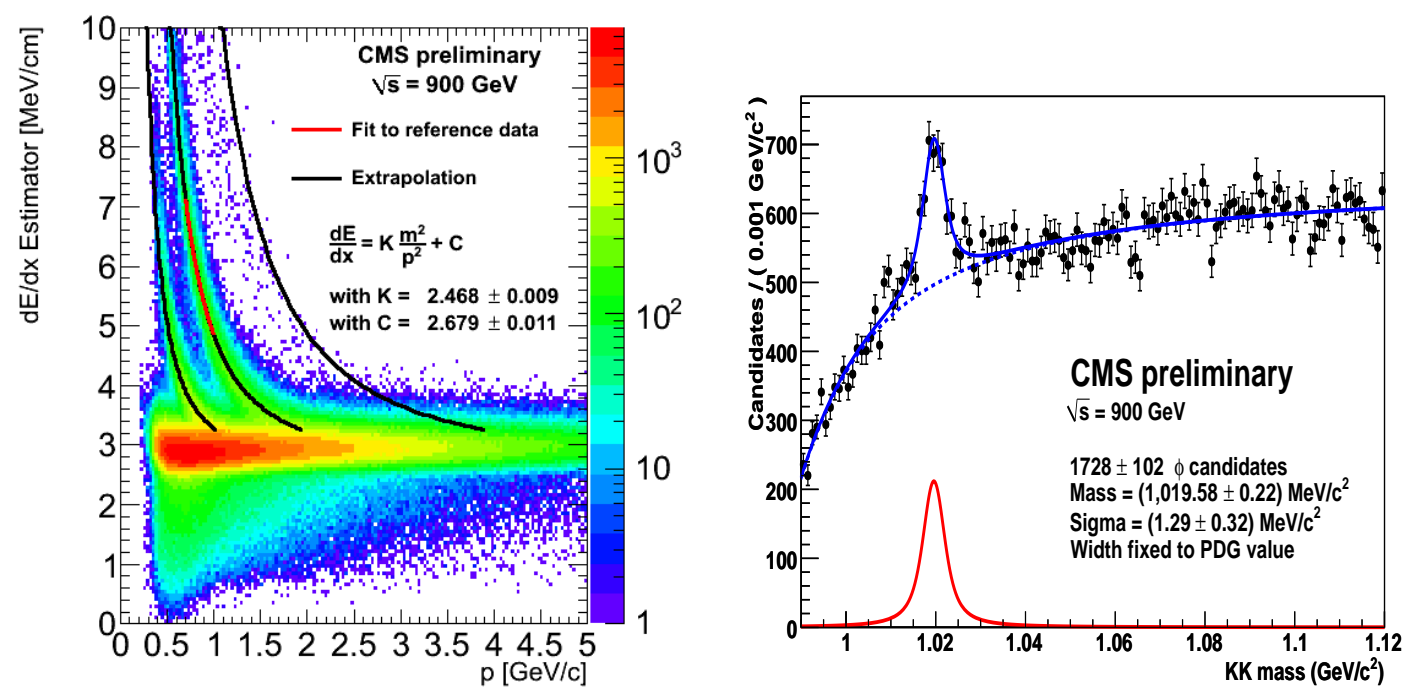

Figure 4: Left: $d E / d x$ versus $p$ in data collected at $0.9 \mathrm{TeV}$, during the December 2009 run. The red line gives a fit with proton mass assumption, in a restricted $p$ range, while black lines show extrapolations. Right: Fit to the mass distribution of kaon candidate pairs in data. The natural width is fixed to the value of $\Gamma_{\phi(1020)}=4.26 \mathrm{MeV} / \mathrm{c}^{2}$.
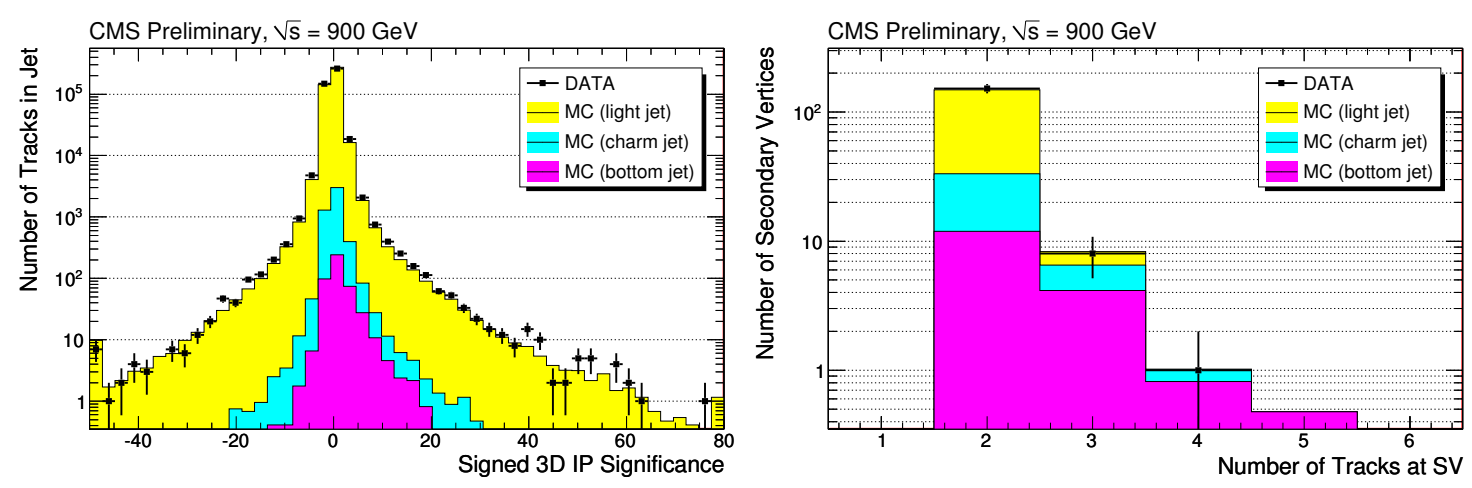

Figure 5: Left: Distribution of the significance of the three-dimensional impact parameter for all tracks in the jet. The data is shown as full circles while the simulated contributions from light flavor, charm and bottom are shown as filled histograms. Right: Number of tracks of the secondary vertex for vertices reconstructed inside jets.

and to achieve better particle identification. In case of HCAL energy calibration the calorimeter cluster energies and the momentum of the corresponding tracks are compared. After calibration the two scale agrees within about 5\% (Fig. 6-left).

In CMS jets can be reconstructed in three ways: using calorimeters only; using calorimeters with track corrections; using the particle flow. The measured and the corresponding simulated jet $p_{T}$ spectra for the three methods are shown in Fig. 7, all of them showing a nice agreement [5].

There are many dijet candidate events identified in the $0.9 \mathrm{TeV}$ sample. The measured distri- 

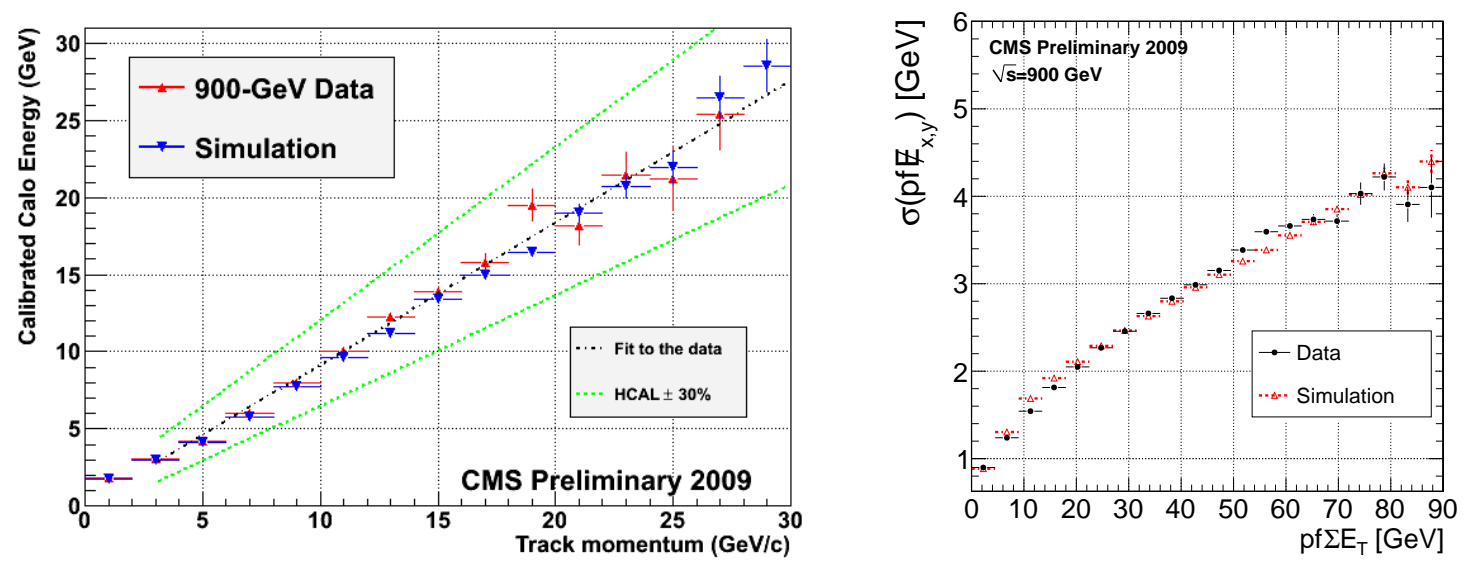

Figure 6: Left: Average calibrated calorimeter response as a function of the track momentum for the $0.9 \mathrm{TeV}$ data and the corresponding simulated minimum bias event sample, integrated over the full tracker acceptance. The dash-dotted line is a linear fit to the data, and the dashed lines show the same fit with a HCAL raw response changed by $\pm 30 \%$, to guide the eye. Right: Particle flow missing $E_{T}$ distribution, after cleanup procedure, in $\sqrt{s}=0.9 \mathrm{TeV}$ data compared with simulation.
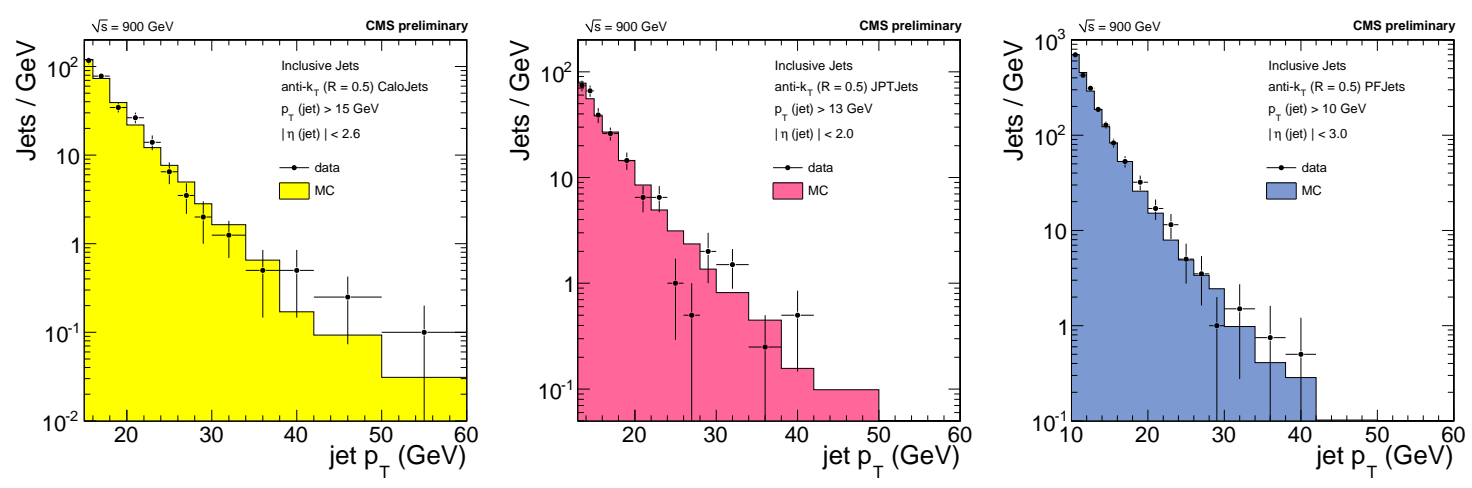

Figure 7: Comparisons of data and simulated inclusive jet $p_{T}$ distributions at $\sqrt{s}=0.9 \mathrm{TeV}$, for calorimeter, track corrected and particle flow jets, respectively.

bution of the number of jet constituents in dijet events matches the simulation expectations.

The measurement of the missing transverse energy proved to be robust over the full data-taking period [6]. Nevertheless very high missing $E_{T}$ values were also detected. They were carefully studied and traced back to anomalous noise in HCAL (functioning of hybrid photo diodes, photomultiplier window hits), in ECAL (single hit crystals, "spikes") and also due to beam-halo muons. A very efficient cleaning procedure was introduced that reduced the missing $E_{T}$ tail.

Here again three reconstruction methods can be compared (Fig. 8). Since no signal is expected yet, the resolution of the measurement can be deduced. With particle flow method the resolution is better than in the calorimeter-only case (Fig. 6-right).

Already with the present dataset nice muon-pair events were identified. 

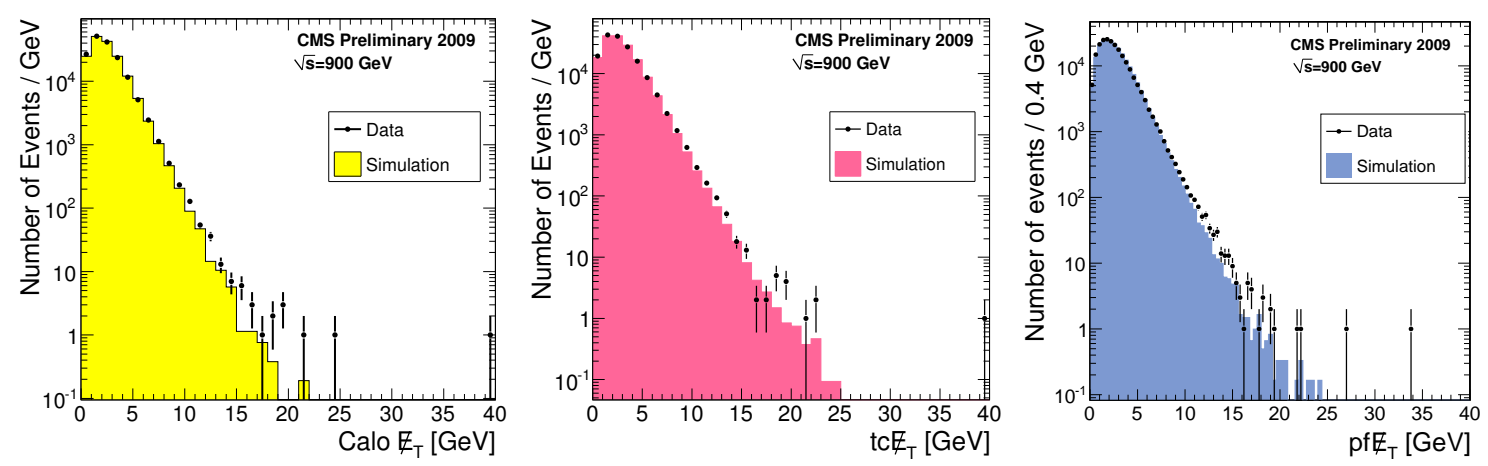

Figure 8: Comparisons of data and simulated missing $E_{T}$ distributions at $\sqrt{s}=0.9 \mathrm{TeV}$, for calorimeter, track corrected and particle flow, respectively.
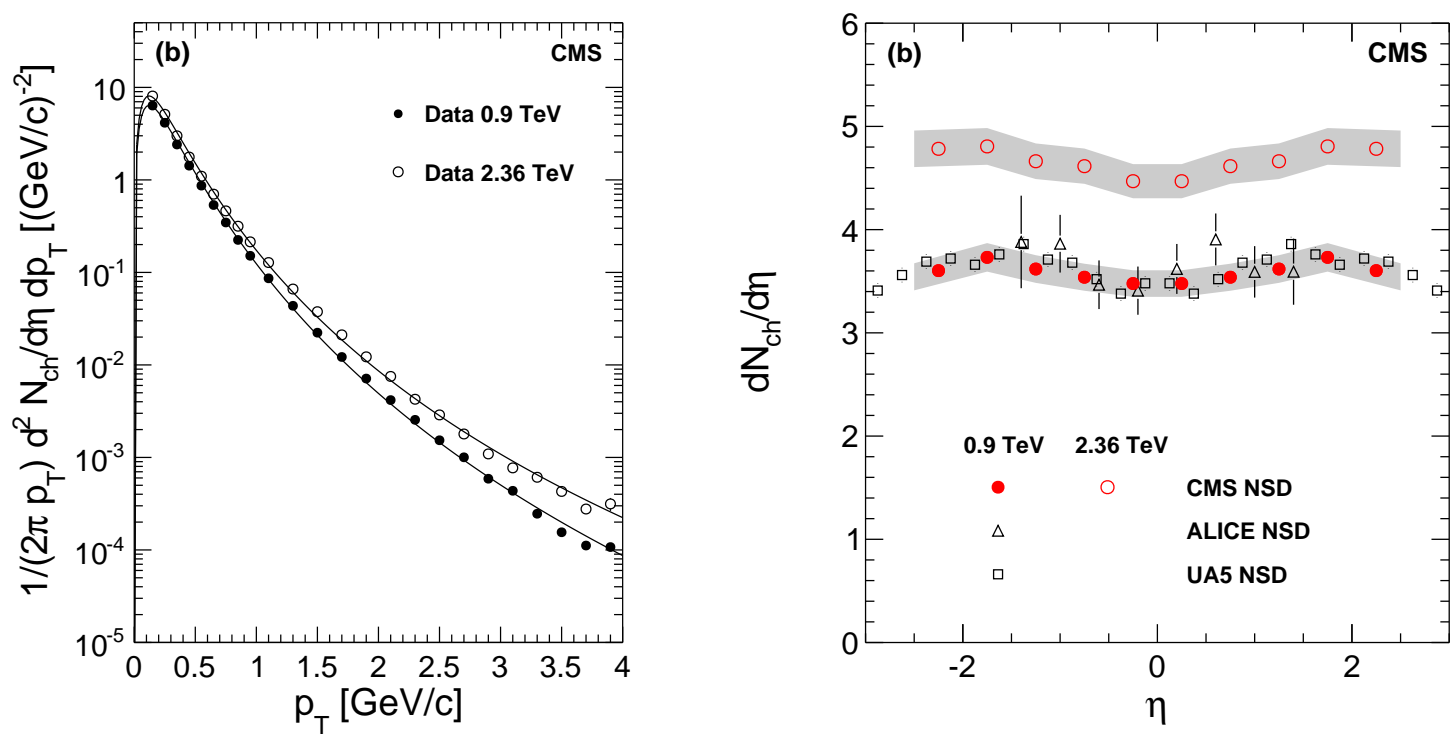

Figure 9: Left: Measured yield of charged hadrons for $|\eta|<2.4$ with systematic uncertainties (symbols), fit with a Tsallis-type function. Right: Reconstructed $d N_{c h} / d \eta$ distributions averaged over the cluster counting, tracklet and tracking methods (circles), compared to data from UA5 and ALICE experiments at $0.9 \mathrm{TeV}$, and the averaged results over the three methods at $2.36 \mathrm{TeV}$ (open circles). The shaded band represents systematic uncertainties of the CMS measurement.

\section{Physics}

In the following three results from the field of soft QCD [8] and forward physics [9] are described.

\subsection{Spectra of charged hadrons}

The measurement of charged hadron spectra is important since it provides basic quantities (yield, average $p_{T}$ ) to study and to tune soft particle production models. It also constitutes the 

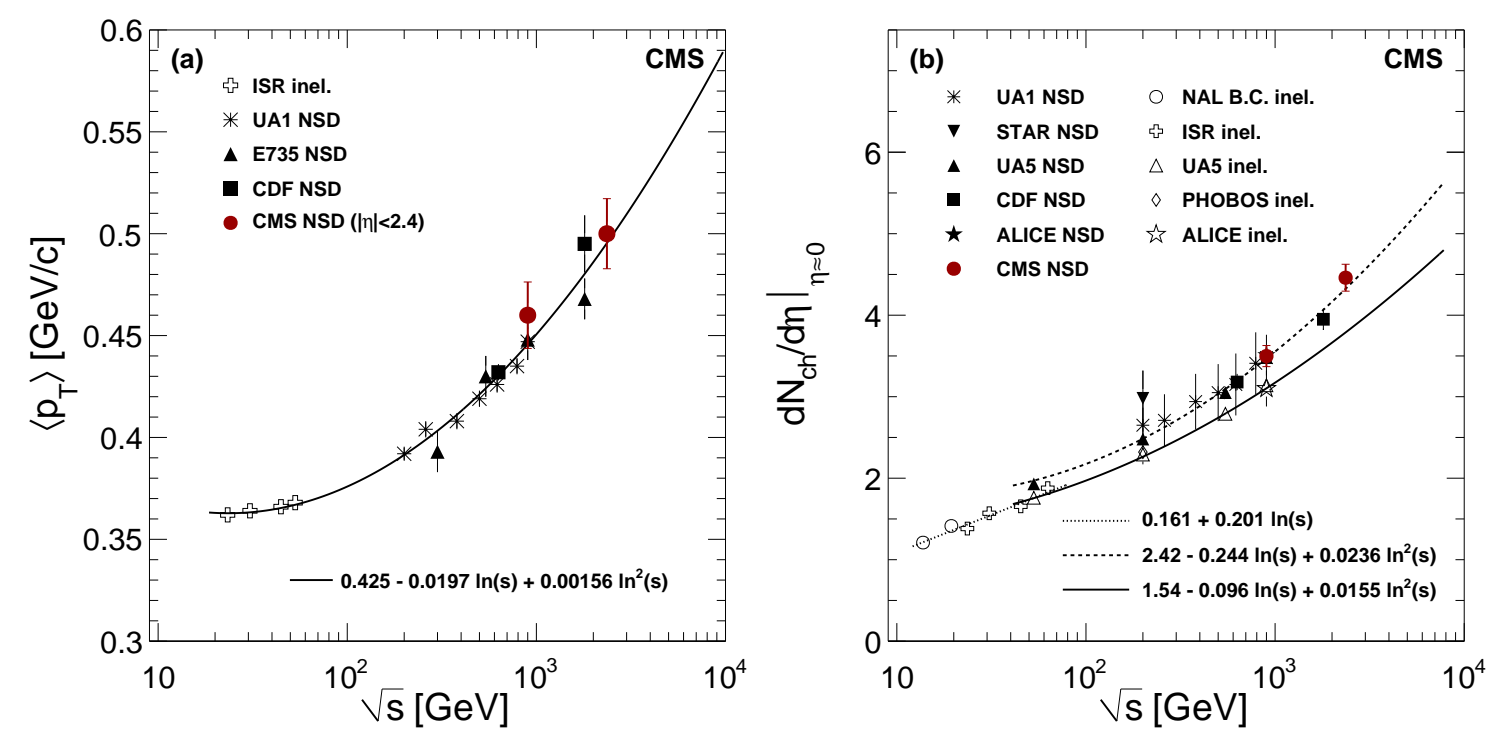

Figure 10: Left: Energy dependence of the average transverse momentum of charged hadrons. The CMS data points are evaluated for the range $|\eta|<2.4$. The curve shows the fit to the data points. The error bars on the CMS data points include systematic uncertainties. Right: Charged-hadron pseudorapidity density in the central region as a function of centre-of-mass energy in $p p$ and $p \bar{p}$ collisions including lower energy data, together with various empirical parameterizations fit to the data corresponding to the inelastic (solid and dotted curves with open symbols) and to the NSD (dashed curve with solid symbols) event selection. The error bars indicate systematic uncertainties, when available.

background of more exclusive processes. In CMS the two-sided scintillator based minimum bias trigger is most sensitive to non-single-diffractive (NSD) collisions, hence all results below were corrected to NSD events. The spectra of hadrons is determined with the pixel and silicon tracker, using three different reconstruction methods: pixel hit counting, tracklets and full tracks [10].

In case of hit counting the number of track hits, clusters, are counted in the barrel region of the pixel detector. With the previously determined collision vertex the pseudorapidity $\eta$ is calculated for each hit, later histogrammed. This very simple method is immune to detector misalignment but it needs several corrections due to looping low- $p_{T}$ particles, decays, $\delta$-electrons, nuclear interactions. It is sensitive even to very low $p_{T}$ particles, of about $30 \mathrm{MeV} / c$. Tracklets are formed out of hit pairs in the pixel detector which are compatible with each other in $\eta$ and azimuth directions. This method has obviously lower background than the previous one, supplemented by a data-driven background subtraction (side-band method). The full tracking method uses all pixel and strip hits left by the charged particle. It provides $\eta$ and $p_{T}$ at the same time. This approach is the most complex one and sensitive to several factors.

The measured $p_{T}$ spectra of charged hadrons at $\sqrt{s}=0.9 \mathrm{TeV}$ and $2.36 \mathrm{TeV}$ in NSD events are shown in Fig. 9-left. A Tsallis-type fit is also displayed: this function successfully combines and describes the low $p_{T}$ exponential and the high $p_{T}$ power law nature of the distribution, with few parameters. The pseudorapidity-density $d N_{c h} / d \eta$ as function of $\eta$ is shown in Fig. 9-right again for both energies, with comparisons to results of other experiments. 

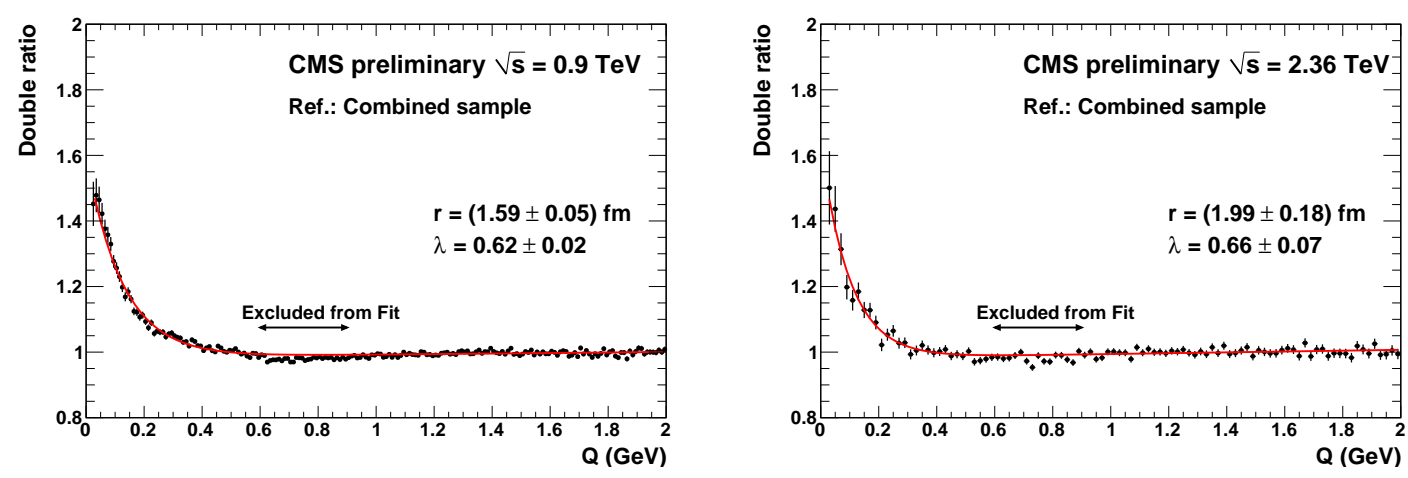

Figure 11: Distributions of the two-particle correlation function for charged hadrons at $\sqrt{s}=0.9 \mathrm{TeV}$ (left) and $2.36 \mathrm{TeV}$ (right) centre-of-mass energies. Measured points (symbols) and the corresponding exponential fits are shown (solid curves).

The centre-of-mass energy dependence of $\left\langle p_{T}\right\rangle$ and $d N_{c h} / d \eta$ at $\eta=0$ is shown in Fig. 10. While the average $p_{T}$ shows the expected rise, the pseudorapidity density gives a higher than expected, accelerated, increase when going from 0.9 to $2.36 \mathrm{TeV}$, if compared to simulation expectations.

\subsection{Bose-Einstein correlation of charged hadrons}

The Bose-Einstein correlation is the manifestation of a quantum effect. It enhances the number of identical boson pairs with low relative momentum. By measuring the ratio of signal and uncorrelated background pairs, as a function of $Q=\sqrt{-\left(p_{1}-p_{2}\right)^{2}}$, the size of the correlated emission region can be inferred. The uncorrelated reference sample is difficult to construct, here a combination of several prescriptions was used. The ratio distribution is in fact the Fourier transform of the original spatial distribution. The distributions are shown in Fig. 11 for $\sqrt{s}=0.9 \mathrm{TeV}$ and $2.36 \mathrm{TeV}$, respectively [7]. The data cannot be fit with a Gaussian function, they rather show an exponential behavior. The extracted radii $r$ and correlation strengths $\lambda$ are shown in the plot.

\subsection{Forward physics}

The energy flow in the forward region is directly sensitive to the amount of parton radiation and to multiparton interaction and provides a complementary measurement to what can be performed in the central region. Ratios of (uncorrected) energy flow between two energies, measured with help of the forward hadronic calorimeter (HF), are given in Fig. 12. These quantities provide valuable input for model tuning in the forward region.

\section{Summary}

The CMS experiment and its detector systems show an excellent performance. Many aspects, physics objects, are already commissioned. The collaboration could produce pioneering results 

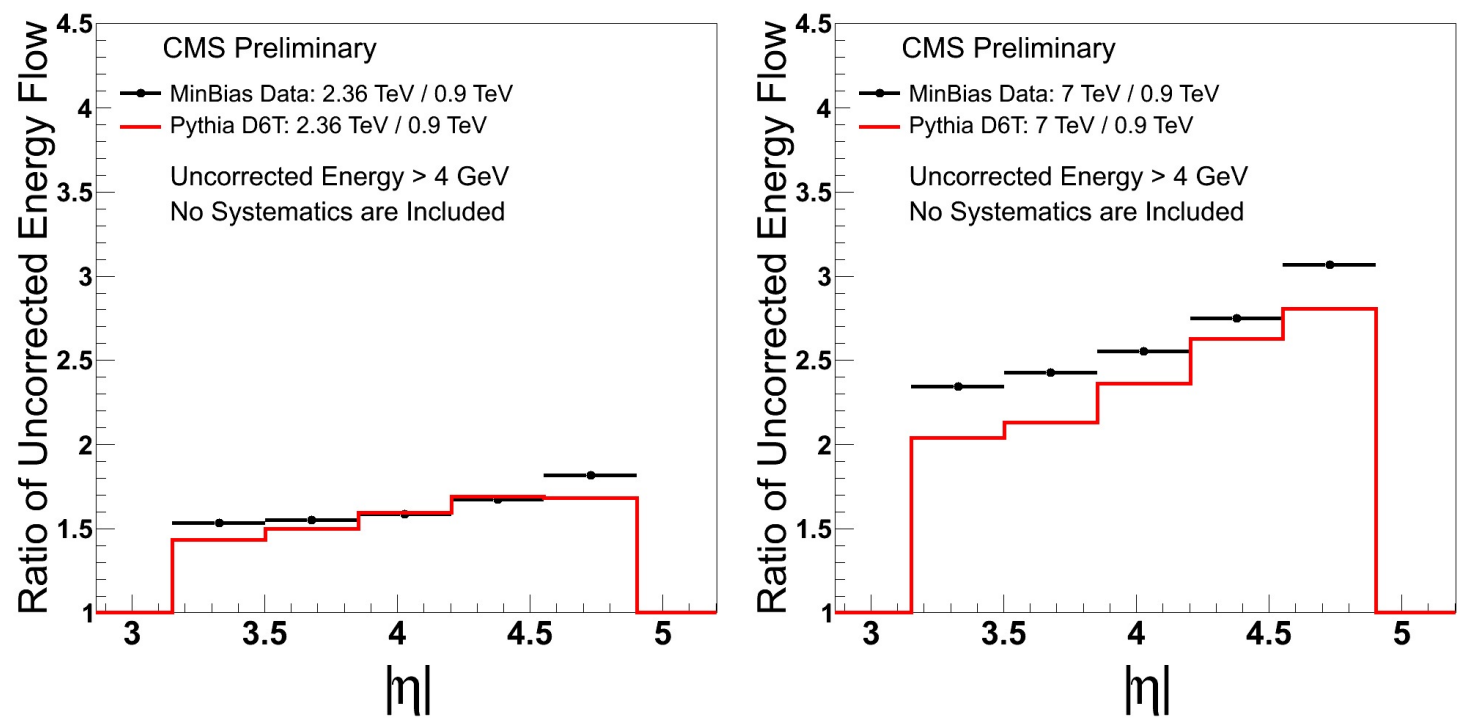

Figure 12: Ratio of energy flow with different collision energies. Systematics are under study, and no correction on energy is applied.

from a small data sample: the first publications using the 0.9 and $2.36 \mathrm{TeV}$ data are already available. This is just the beginning of a successful physics program at the LHC, with possible future discoveries.

\section{Acknowledgments}

The author wishes to thank to the Hungarian Scientific Research Fund (K 81614, NK 81447) and the Swiss National Science Foundation (128079) for support.

\section{References}

[1] R. Adolphi et al. [CMS Collaboration], The CMS experiment at the CERN LHC, JINST 3 (2008) S08004.

[2] CMS Collaboration, Electromagnetic physics objects commissioning with first LHC data, CMS PAS EGM-10-001.

[3] CMS Collaboration, Tracking and Vertexing Results from First Collisions, CMS PAS TRK-10-001.

[4] CMS Collaboration, Commissioning of the Particle-flow Event Reconstruction with the first LHC collisions recorded in the CMS detector, CMS PAS PFT-10-001.

[5] CMS Collaboration, Jets in 0.9 and $2.36 \mathrm{TeV}$ pp Collisions, CMS PAS JME-10-001.

[6] CMS Collaboration, Missing $E_{T}$ in 0.9 and $2.36 \mathrm{TeV}$ pp Collisions, CMS PAS JME-10-002.

[7] CMS Collaboration, Measurement of Bose-Einstein correlations with first CMS data, CMS PAS QCD-10-003.

[8] K. Krajczár et al. [CMS Collaboration], QCD physics from CMS, in these proceedings. 
[9] S. Sen et al. [CMS Collaboration], Measurement of the transverse energy flow and forward jets, in these proceedings.

[10] V. Khachatryan et al. [CMS Collaboration], Transverse momentum and pseudorapidity distributions of charged hadrons in pp collisions at sqrt( $s)=0.9$ and $2.36 \mathrm{TeV}$, JHEP 02 (2010) 041 [arXiv:1002.0621 [hep-ex]]. 